\title{
Aperiodic variability of low-mass X-ray binaries at very low frequencies
}

\author{
P. Reig ${ }^{1,3}$, I. Papadakis ${ }^{1,2}$, and N. D. Kylafis ${ }^{1,2}$ \\ 1 Foundation for Research and Technology-Hellas, 71110 Heraklion, Crete, Greece \\ 2 Physics Department, University of Crete, PO Box 2208, 71003 Heraklion, Crete, Greece \\ ${ }^{3}$ G.A.C.E, Departament d'Astronomia i Astrofísica, Universitat de Valéncia, 46071 Paterna-Valencia, Spain
}

Received 31 July 2002 / Accepted 13 November 2002

\begin{abstract}
We have obtained discrete Fourier power spectra of a sample of persistent low-mass neutron-star X-ray binaries using long-term light curves from the All Sky Monitor on board the Rossi X-ray Timing Explorer. Our aim is to investigate their aperiodic variability at frequencies in the range $1 \times 10^{-7}-5 \times 10^{-6} \mathrm{~Hz}$ and compare their properties with those of the black-hole source Cyg X-1. We find that the classification scheme that divides LMXBs into $\mathrm{Z}$ and atoll sources blurs at very low frequencies. Based on the long-term ( years) pattern of variability and the results of power-law fits $\left(P(v) \propto v^{-\alpha}\right)$ to the $1 \times 10^{-7}-5 \times 10^{-6} \mathrm{~Hz}$ power density spectra, low-mass neutron-star binaries fall into three categories. Type I includes all $\mathrm{Z}$ sources, except Cyg X-2, and the atoll sources GX9+1 and GX13+1. They show relatively flat power spectra $(\alpha \lesssim 0.9)$ and low variability (rms $\lesssim 20 \%$ ). Type II systems comprise 4U 1636-53, 4U 1735-44 and GX3+1. They are more variable $(20 \% \lesssim \mathrm{rms} \lesssim 30 \%)$ and display steeper power spectra $(0.9 \lesssim \alpha \lesssim 1.2)$ than type I sources. Type III systems are the most variable $(\mathrm{rms}>30 \%)$ and exhibit the steepest power spectra $(\alpha>1.2)$. The sources 4U 1705-44, GX354-0 and 4U 1820-30 belong to this group. GX9+9 and Cyg X-2 appear as intermediate systems in between type I and II and type II and III sources, respectively. We speculate that the differences in these systems may be caused by the presence of different types of mass-donor companions. Other factors, like the size of the accretion disc and/or the presence of weak magnetic fields, are also expected to affect their low-frequency X-ray aperiodic varibility.
\end{abstract}

Key words. stars: neutron - binaries: close - X-rays: binaries - accretion: accretion discs

\section{Introduction}

A low-mass X-ray binary (LMXB) contains a neutron star which is accreting material via Roche lobe overflow from a companion star of spectral type later than A. Due to the high angular momentum of the accretion flow an accretion disc is formed around the compact object (see e.g. White 1989). Unlike high-mass X-ray binaries, whose optical spectrum is dominated by the emission from the massive companion, in LMXBs the mass-losing star is usually not seen owing to the contribution of the accretion disc (Cowly et al. 1991; Shahbaz et al. 1996). Lewin \& van Paradijs (1985) proposed that the different classes of LMXBs might reflect the type of companion, with the brighter systems (usually those located in the galactic bulge) containing an evolved star and the fainter systems a main-sequence star. Based on infrared spectroscopic observations, Bandyopadhyay et al. (1999) suggested that not only the luminosity class but also the spectral type may be different.

Various schemes have been proposed to categorise the LMXBs: X-ray spectral behaviour as a function of intensity

Send offprint requests to: P. Reig,

e-mail: pablo@physics.uoc.gr
(Parsignault \& Grindlay 1978), cluster analysis of a large number of source characteristics (Ponman 1982), detailed X-ray spectral fits (White \& Mason 1985), X-ray hardness-intensity and colour-colour diagrams (Schulz et al. 1988), age and location in the Galaxy (Naylor \& Podsiadlowski 1993). In general, a bimodal distribution of sources and a correlation with luminosity is found.

More relevant for the purpose of this paper is the classification scheme in terms of the rapid aperiodic variability and the patterns that these sources display in X-ray colour-colour diagrams (Hasinger \& van der Klis 1989). In this scheme LMXBs are divided into two different subclasses, known as $\mathrm{Z}$ and atoll sources. In the frequency range $10^{-2}-10^{3} \mathrm{~Hz}$ the power spectra of atoll and $\mathrm{Z}$ sources are represented by a power law with index 1-1.5 (atoll sources) and 1.5-2 ( $\mathrm{Z}$ sources), which describes the spectrum at low frequencies (below $\sim 1 \mathrm{~Hz}$ ) and a power law plus exponential cut off with index $0-0.8$ (atoll sources), $\sim 0$ (Z sources) and $v_{\text {cut }}=0.3-25 \mathrm{~Hz}$ (atoll sources), $v_{\mathrm{cut}}=30-100 \mathrm{~Hz}$ ( $\mathrm{Z}$ sources) at higher frequencies (van der Klis 1995 and references therein). The strength of these two components correlates with the position of the source in the colour-colour diagram. On top of this continuum 
Table 1. List of sources.

\begin{tabular}{|c|c|c|c|c|c|c|c|c|c|c|}
\hline $\begin{array}{l}\text { Source } \\
\text { number }\end{array}$ & $\begin{array}{l}\text { Source } \\
\text { name }\end{array}$ & $\begin{array}{l}\text { Alternative } \\
\text { name }\end{array}$ & Class $^{a}$ & $\begin{array}{c}L_{\mathrm{X}}^{b} \times 10^{38} \\
\mathrm{erg} \mathrm{s}^{-1}\end{array}$ & $I_{\mathrm{m}}^{e}$ & $\Delta I^{f}$ & $\alpha^{g}$ & $\chi^{2}$ d.o.f. $^{h}$ & $\mathrm{rms}^{i}$ & $\begin{array}{c}P_{\text {orb }}^{j} \\
\mathrm{hr}\end{array}$ \\
\hline \multicolumn{11}{|c|}{ Type I } \\
\hline 1 & $4 U$ 1617-15 & Sco X-1 & $\mathrm{Z},-, \mathrm{I}, 2, \mathrm{~B}$ & 0.7 & 920 & 2.1 & $0.67 \pm 0.06$ & $23 / 24$ & $14.4 \pm 0.2$ & \multirow[t]{2}{*}{18.9} \\
\hline 2 & 4U 1642-45 & GX $340+0$ & $\mathrm{Z},-, \mathrm{II},-, \mathrm{B}$ & 2.2 & 30 & 2.0 & $0.63 \pm 0.05$ & $43 / 24$ & $13.8 \pm 0.2$ & \\
\hline 3 & $4 \mathrm{U} 1702-36$ & GX 349+2 & $\mathrm{Z}, \mathrm{Sp}, \mathrm{I}, 2, \mathrm{~B}$ & 0.7 & 52 & 2.0 & $0.35 \pm 0.06$ & $21 / 24$ & $15.6 \pm 0.3$ & \multirow[t]{2}{*}{$21.8-22.5$} \\
\hline 4 & 4U 1758-25 & GX 5-1 & $\mathrm{Z}, \mathrm{Sp}, \mathrm{I},-, \mathrm{B}$ & 4.0 & 73 & 1.6 & $0.73 \pm 0.05$ & $48 / 23$ & $12.9 \pm 0.2$ & \\
\hline 5 & 4U 1758-20 & GX 9+1 & $\mathrm{A}, \mathrm{Sp}, \mathrm{I}, 2, \mathrm{~B}$ & 1.2 & 40 & 1.7 & $0.58 \pm 0.10$ & $35 / 23$ & $10.5 \pm 0.2$ & \multirow{3}{*}{592.8} \\
\hline 6 & 4U 1811-17 & GX 13+1 & $\mathrm{A}, \mathrm{Sp}, \mathrm{I},-, \mathrm{B}$ & 0.8 & 23 & 2.0 & $0.63 \pm 0.06$ & $57 / 23$ & $11.6 \pm 0.2$ & \\
\hline 7 & 4U 1813-14 & GX 17+2 & Z,Sp,I,2,B & 2.3 & 47 & 1.4 & $0.51 \pm 0.06$ & $31 / 24$ & $11.7 \pm 0.2$ & \\
\hline 8 & 4U 1728-16 & GX 9+9 & $\mathrm{A}, \mathrm{Su}, \mathrm{I},-, \mathrm{B}$ & 0.3 & 17 & 1.5 & $0.85 \pm 0.11$ & $35 / 23$ & $10.3 \pm 0.2$ & 4.2 \\
\hline \multicolumn{11}{|c|}{ Type II } \\
\hline 9 & $4 U$ 1636-53 & & $\mathrm{A}, \mathrm{Su}, \mathrm{II}, 1, \mathrm{D}$ & 0.2 & 14 & 4.0 & $0.97 \pm 0.07$ & $35 / 24$ & $25.9 \pm 0.5$ & 3.8 \\
\hline 10 & 4U 1735-44 & & $\mathrm{A}, \mathrm{Su}, \mathrm{II},-, \mathrm{D}$ & 0.4 & 12 & 2.4 & $0.85 \pm 0.07$ & $51 / 24$ & $22.4 \pm 0.4$ & 4.65 \\
\hline 11 & 4U 1744-26 & GX $3+1$ & $\mathrm{~A}, \mathrm{Sp}, \mathrm{I},-, \mathrm{B}$ & 0.3 & 21 & 3.0 & $1.13 \pm 0.10$ & $21 / 23$ & $22.8 \pm 0.5$ & \\
\hline 12 & 4U 2142+38 & Cyg X-2 & $\mathrm{Z},-, \mathrm{I},-, \mathrm{B}$ & 1.4 & 38 & 3.7 & $1.17 \pm 0.07$ & $67 / 25$ & $24.1 \pm 0.4$ & 236 \\
\hline \multicolumn{11}{|c|}{ Type III } \\
\hline 13 & $4 \mathrm{U} 1705-44$ & \multirow{3}{*}{ GX 354-0 } & $\mathrm{A}, \mathrm{Su},-, 2, \mathrm{D}$ & 0.13 & 13 & 30 & $1.22 \pm 0.12$ & $76 / 24$ & $67.5 \pm 1.2$ & \\
\hline 14 & 4U 1728-33 & & $\mathrm{A}, \mathrm{Su}, \mathrm{II}, 1, \mathrm{D}$ & $0.1^{c}$ & 6 & 7.5 & $1.79 \pm 0.11$ & $55 / 24$ & $41.7 \pm 0.8$ & \\
\hline 15 & 4U 1820-30 & & $\mathrm{A}, \mathrm{Su}, \mathrm{II},-,--$ & 0.6 & 19 & 6.0 & $1.59 \pm 0.11$ & $34 / 24$ & $32.4 \pm 0.6$ & 0.19 \\
\hline \multicolumn{11}{|c|}{ Other sources } \\
\hline 16 & $4 \mathrm{U} 1956+35$ & Cyg X-1 & $\mathrm{BH}$ & $0.7^{d}$ & 30 & 10 & $1.04 \pm 0.11$ & $129 / 25$ & $54.0 \pm 0.9$ & 134.4 \\
\hline \multicolumn{11}{|c|}{${ }^{a}$ Classification of the system in the various schemes (see text): $\mathrm{Z}, \mathrm{A}=$ atoll, $\mathrm{Sp}=$ supercritical, $\mathrm{Su}=$ subcritical } \\
\hline \multicolumn{11}{|c|}{$\mathrm{B}=$ bulge, $\mathrm{D}=$ disc, $\mathrm{I}=$ class $\mathrm{I}, \mathrm{II}=$ class II, $1=$ one spectral component $2=$ two spectral components $\mathrm{BH}=$ black-hole source. } \\
\hline \multicolumn{11}{|c|}{${ }^{b}$ X-ray luminosity, from Christian \& Swank (1997) in the energy range $0.5-20 \mathrm{keV}$. } \\
\hline \multicolumn{11}{|c|}{${ }^{c}$ From Narita et al. (2001) in the $1-10 \mathrm{keV}$ bandwidth. } \\
\hline \multicolumn{11}{|c|}{${ }^{d}$ From Belloni et al. (1996) in the $1-30 \mathrm{keV}$ bandwidth. } \\
\hline \multicolumn{11}{|c|}{${ }^{e}$ Mean ASM count $\mathrm{s}^{-1}$ in the energy range $1.3-12.2 \mathrm{keV}$. } \\
\hline \multicolumn{11}{|c|}{${ }^{f} \Delta I=I_{\max } / I_{\min }}$. \\
\hline \multicolumn{11}{|c|}{${ }^{g}$ Best-fit power-law index of the power spectra. Errors are the $1 \sigma$ confidence interval. } \\
\hline \multicolumn{11}{|c|}{${ }^{h} \chi^{2}$ value and number of degrees of freedom of the fits to the power density spectra. } \\
\hline \multicolumn{11}{|c|}{${ }^{i}$ Fractional (\%) rms amplitude obtained from the light curves as the ratio of the variance over the mean. } \\
\hline
\end{tabular}

several types of quasi-periodic oscillations are seen (van der Klis 1994a, 1994b; Wijnands \& van der Klis 1999; Belloni et al. 2002). On long time scales ( $>$ few tens of days) the studies on LMXB have concentrated on the search for periodicities (Smale \& Lochner 1992; Kong et al. 1998). In this work we investigate the long-term aperiodic variability of 9 atoll and $6 \mathrm{Z}$ persistent LMXBs by comparing the characteristics of their power density spectra in the frequency range $1 \times 10^{-7}-5 \times 10^{-6} \mathrm{~Hz}$ and the patterns of variability of their light curves.

\section{Data analysis}

We have analysed the light curves obtained by the All Sky Monitor (ASM) on board the Rossi X-ray Timing Explorer (RXTE) of all persistent neutron-star systems showing an average count rate above $5 \mathrm{c} / \mathrm{s}$ (Table 1 ). The data were retrieved from the Definitive Products Database. The time span by the observations is about 5.7 years, from February 1996 to October 2001 (JD 2450130-2452200). The ASM consists of three wide-angle $\left(6^{\circ} \times 90^{\circ}\right)$ shadow cameras (SSC1-3) equipped with position-sensitive xenon proportional counters with a total collecting area of $90 \mathrm{~cm}^{2}$. The ASM scans $\sim 80 \%$ of the sky every $\sim 90$ min in a series of dwells of about $90 \mathrm{~s}$ each. Any given $\mathrm{X}$-ray source is observed in about 5-10 dwells every day. The ASM is sensitive to X-rays in the energy band $1.3-12.2 \mathrm{keV}$. For more information on the ASM see Levine et al. (1996).

In order to estimate the power density spectrum (PDS) of each source, we used 1-day binned light curves. Gaps due to detector failure or lack of data were filled by linear interpolation, adding appropriate random noise. In order to avoid having a strong dependence on interpolation, which might introduce undesired features in the power spectrum, data interpolation was done only when the number of consecutive missing points was smaller than $2 \%$ of the total number of points in the light curve. These short gaps are randomly distributed over the whole light curve. The average number of missing points per gap was typically 2.5 for the 1-day rebinned light curves.

The long gaps in the light curves, i.e. those occurring when no data points exist during an interval of time longer than $2 \%$ of the total length of the observation, divide the light curve into 


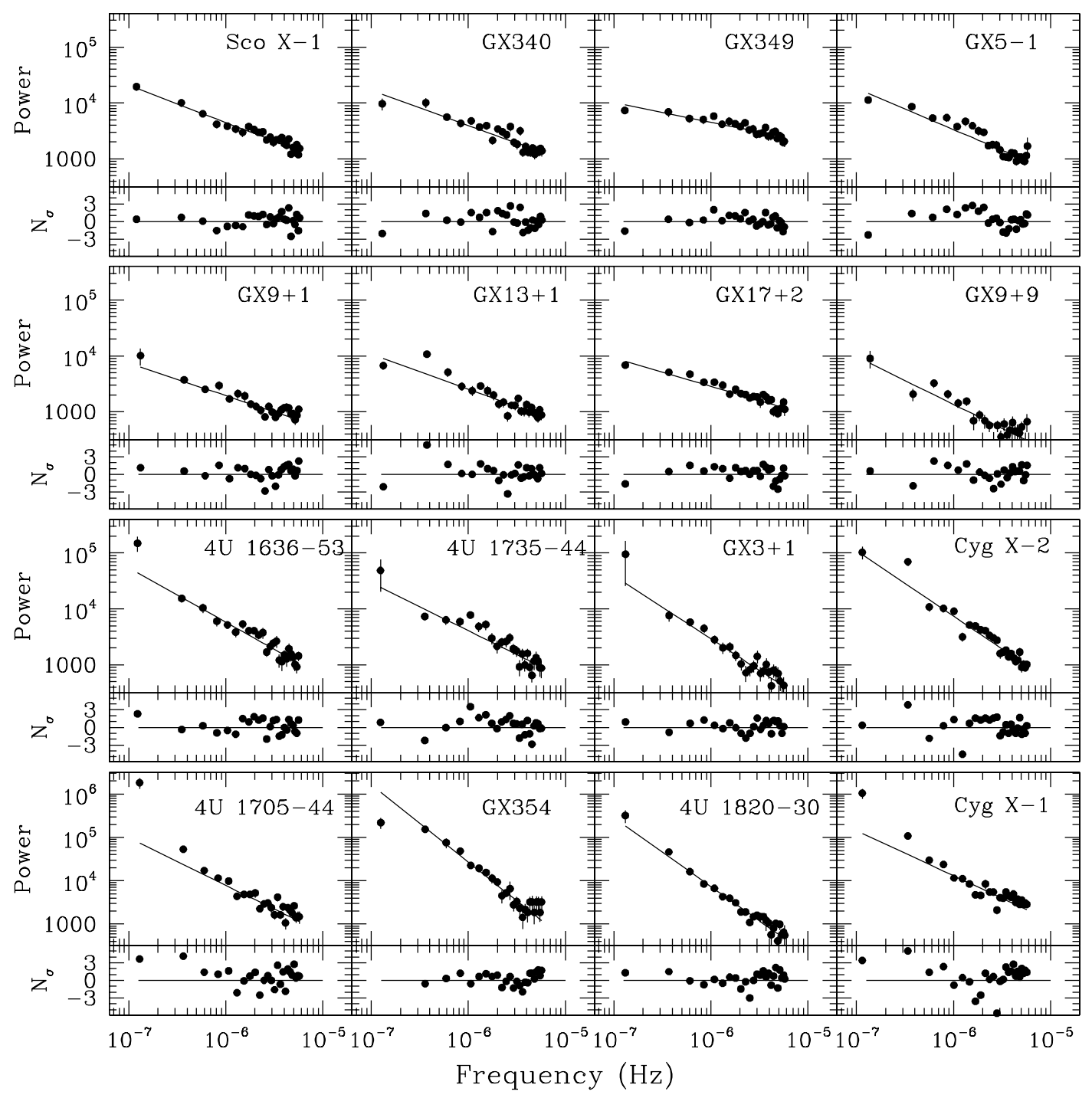

Fig. 1. Power density spectra of the sources studied in this work and the best-fit power law. The residuals represent the difference between the observational points and the model, expressed as number of $\sigma$.

segments of different duration. In order to extract the maximum amount of information from the light curves we used the discrete (slow) Fourier transform on each observational segment of data. Since the data segments had different lengths, the resulting power density spectra covered different frequency ranges. The PDS of the individual segments were merged together, sorted in order of increasing frequency, and rebinned in frequency to have at least 40 points per bin. The PDS were white noise subtracted and normalized such that their integral over a frequency range $v_{1}-v_{2}$ gives the squared fractional rms variability of the light curves due to variations on time scales from $v_{2}^{-1}$ to $v_{1}^{-1}$ (Belloni \& Hasinger 1990; Miyamoto et al. 1991).

\section{Results}

Figure 1 shows the power density spectra (PDS) of all the sources studied in this work, together with the best-fit powerlaw model and residuals. The residuals represent the number of $\sigma$ that the observations deviate from the model. For the sake of comparison we have also analysed the ASM light curve of the black-hole source Cyg X-1 in the same way (for details on the aperiodic variability of $\mathrm{Cyg} X-1$ at very low frequencies see Reig et al. 2002). All sources show a red-noise dominated power density spectrum. No flattening at low frequencies is seen in any of the PDS. Although a single power law did not give acceptable fits in some cases, it provides a convenient way to compare the variability of the spectral parameters among the different types of systems. Characterising the LMXBs in terms of the shape of the power density spectrum alone may give rise to ambiguities since intrinsically different time series can produce similar power density spectra (e.g. Press 1978). That is to say, two sources may display the same PDS even though their variability mechanism may not be the same. Therefore we have also examined the X-ray variability of the sources in the time domain by obtaining the root mean square (rms) and the intensity distribution function (IDF). The rms amplitude gives a measure of the variability of the source and was computed from the light curve as $\mathrm{rms}^{2}=\sigma^{2} / \bar{x}^{2}$, where $\bar{x}$ is the mean count rate and $\sigma^{2}=\sigma_{\text {obs }}^{2}-\sigma_{\exp }^{2}$ is the difference between the 


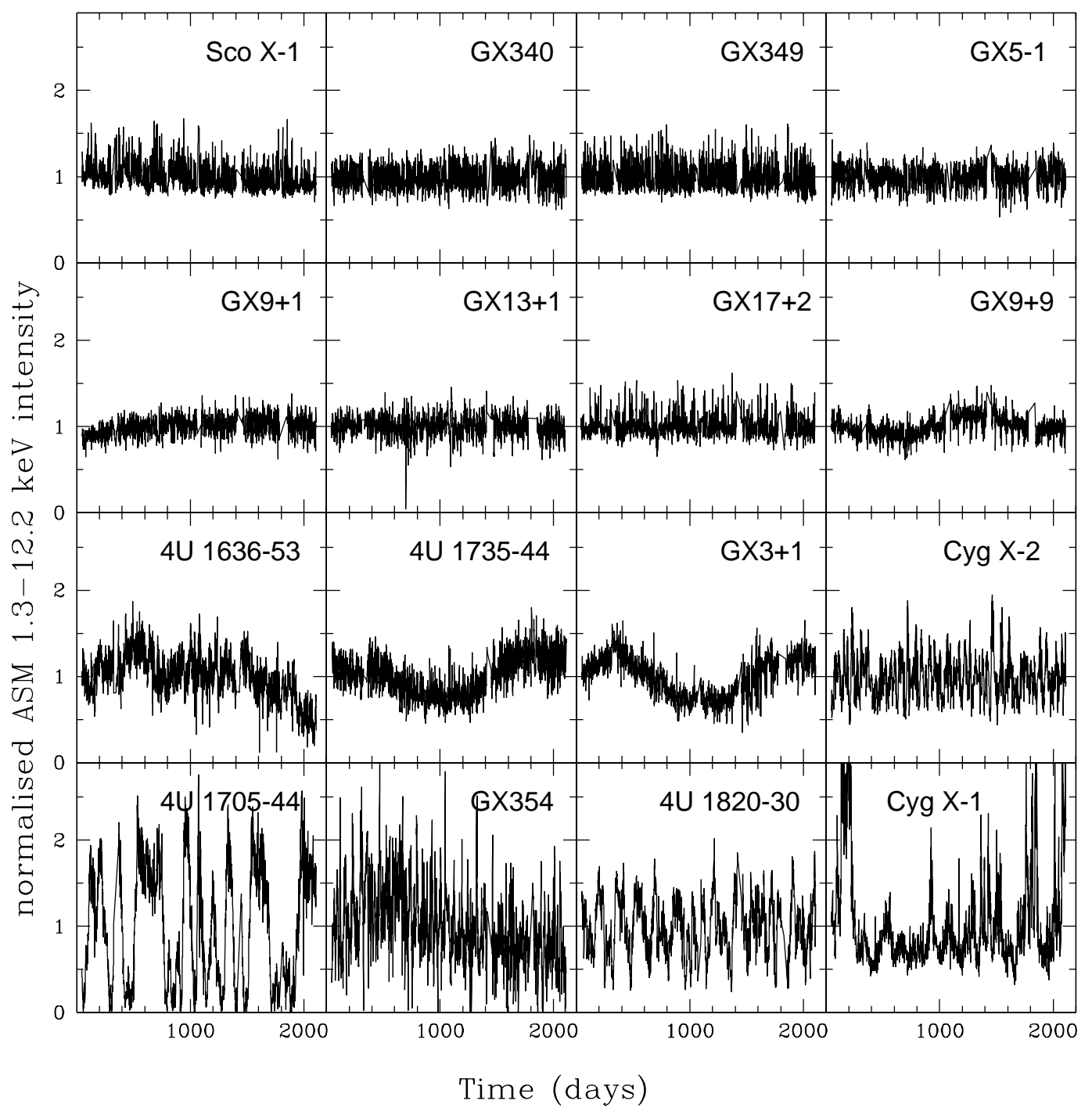

Fig. 2. ASM light curves of the sources studied in this work. Each point represents 1 day.

observed variance, $\sigma_{\text {obs }}^{2}=\sum_{i}\left(x_{i}-\bar{x}\right)^{2} / N$, and the expected variance, $\sigma_{\exp }^{2}=\sum_{i} \sigma_{i}^{2} / N\left(\sigma_{i}\right.$ are the experimental errors, and $N$ is the total number of points). Figure 2 shows the light curves of the sources analysed here. The IDFs (Fig. 3) show how often a given (normalised) intensity occurs. The abscissa represents the intensity of the source normalised to its mean value in steps of 0.1 , whereas the ordinate gives the number of times (i.e. the frequency) that certain values of the normalised intensity occur. The IDF plots also give a measure of the source variability amplitude. Narrow IDFs indicate that the source is roughly stable, showing low-amplitude variations, whereas highly variable sources will show many populated bins in their IDF plots, hence resulting in wide IDFs or narrow IDFs with extended tails.

Figure 4 shows the rms of the light curves and the bestfit PDS power-law indices. By comparing these power spectral and timing parameters LMXBs can be divided into three groups. The first group includes all $\mathrm{Z}$ sources, excluding Cyg X-2, plus the atoll sources GX13+1, GX9+1 and GX9+9. These are bright sources showing low-amplitude and fast variations (on time scales of $\sim 1-2$ days). As a result, their rms values are small, their PDSs are flat and their IDFs appear to be narrow and tend to display positive tails. The second group comprises the atoll sources $4 \mathrm{U}$ 1636-53, 4U 1735-44 and GX3+1 and the $\mathrm{Z}$ source Cyg X-2. These sources show similar ASM light curves as the previous group (i.e. low-amplitude variations on time scales of $\sim 1-2$ days) but with an extra long-term modulation. This modulation produces more power at low frequencies giving rise to steeper power spectra and increasing their rms variability. Their IDFs are also broader when compared to the IDFs of the sources in the previous group and tend to exhibit negative tails. The third group contains those systems that cannot be placed in neither of the two previous groups, namely 4U 1705-44, 4U 1820-30 and GX354. Although they do not form as homogeneous a group as type I and II sources they display the highest amplitude variations, the steepest power spectra, and the broadest IDFs. Quasi-periodic oscillations seem to be a characterising feature of this group. For the purpose of discussion we will refer to these groups as type I, type II and type III, respectively.

For comparison, all figures include data of the black-hole source Cyg X-1. Its PDS slope agrees with that of type II 


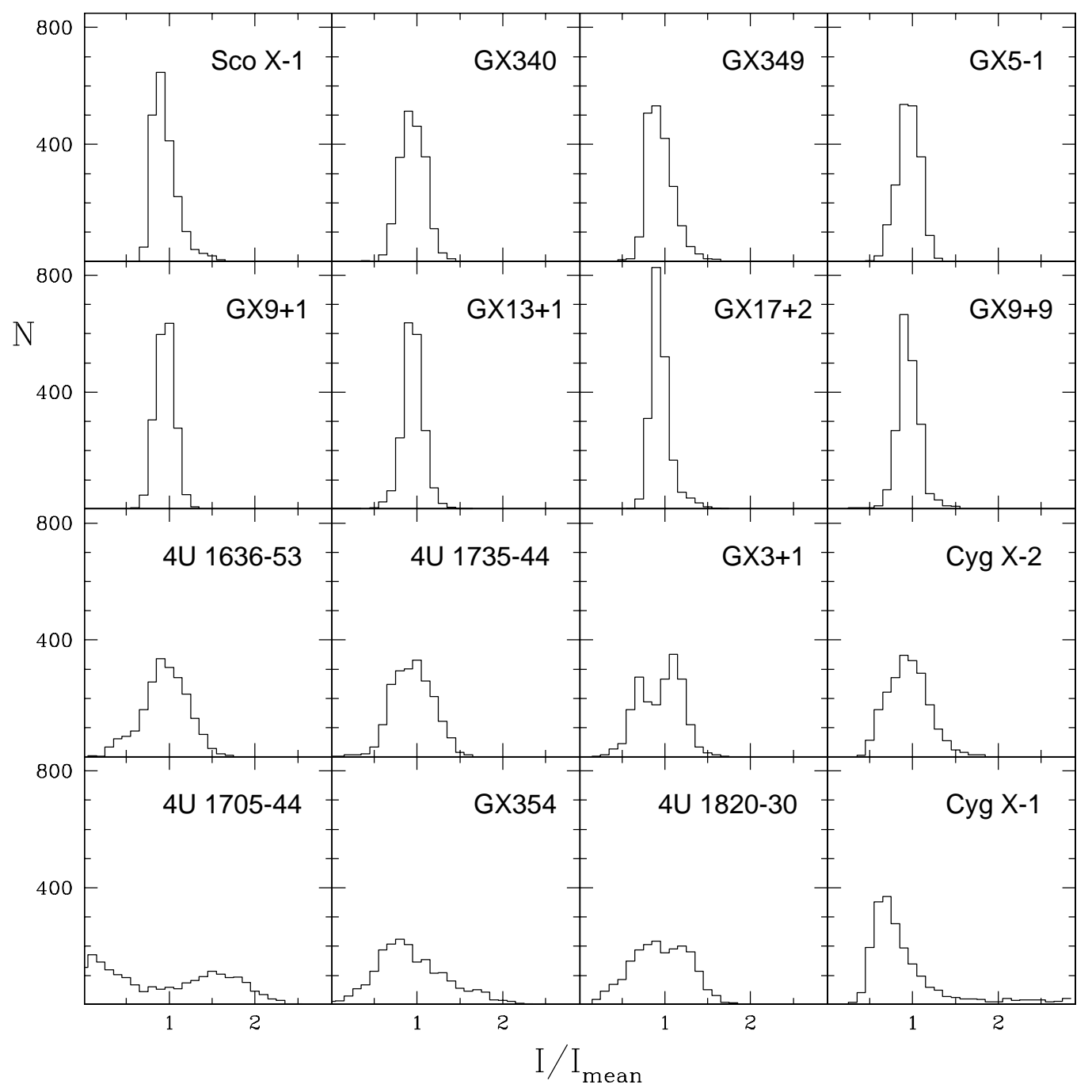

Fig. 3. Distribution of the ASM intensity (normalised to the mean value) of the sources studied in this work.

objects, although it is also consistent with that of the type III source 4U 1705-44. Its light curve and IDF are, however, rather peculiar (note the long tail above $I / I_{\text {mean }}=1.5$ ).

Since sources belonging to the same group show very similar characteristics we have obtained a mean power density spectrum for each group by averaging the PDS of the individual sources pertaining to that group (Fig. 5). The PDS of Cyg X-1 has also been plotted (filled circles). Each group can then be distinguished on the basis of the slope of the PDS and the amplitude of variability. The power-law index $\alpha$ and the fractional amplitude of variability rms calculated by integrating over the power-law model in the frequency range $1 \times 10^{-7}-5 \times 10^{-6} \mathrm{~Hz}$ of the mean PDS for each group of systems are: $\alpha=0.62 \pm 0.03$, $\mathrm{rms}=11.5 \pm 0.3 \%$ for type I sources; $\alpha=1.09 \pm 0.05$, $\mathrm{rms}=15.0 \pm 0.4 \%$ for type II sources and $\alpha=1.61 \pm 0.08$, $\mathrm{rms}=29.5 \pm 0.6 \%$ for type III sources.

Type I and type II systems exhibit similar PDS, except for the fact that type II sources contain less (more) power at higher (lower) frequencies than the type I systems, which agrees with the characteristic shape of the light curves, i.e. fast variations in type I LMXB and long-term trends in type II LMXB. Type III power spectra show the largest amplitude variations at all frequencies (except at the highest ones). This again is in agreement with the flaring like appearance of their light curves.

Finally, we find a correlation between source luminosity, $L_{\mathrm{X}}$, and $\mathrm{rms}$, in the sense that more luminous systems tend to be less variable. In Fig. 6, we plot the rms vs. source luminosity. An anticorrelation between these two quantities is apparent in this figure. This result is verified when we used the Kendall's $\tau$ nonparametric statistic (Press et al. 1992) in order to investigate, quantitatively, whether the apparent anticorrelation between the two quantities is significant or not. We find $\tau=-0.56$; the probability that we would get this value by chance if $L_{\mathrm{X}}$ and $\mathrm{rms}$ were uncorrelated is $\sim 3.6 \times 10^{-3}$. We fitted a straight line to the $\log \left(L_{X}\right)-\log (\mathrm{rms})$ plot using the least-squares bisector line method (Isobe et al. 1990). The results show that $\mathrm{rms} \propto L_{\mathrm{X}}^{-\beta}$, with $\beta=0.6 \pm 0.1$.

\section{Discussion}

We have investigated the aperiodic X-ray variability of all persistent neutron-star systems showing an average count rate above 5c/s (ASM RXTE) over a period spanning about 5.7 years. A new scheme is proposed in which LMXBs can 


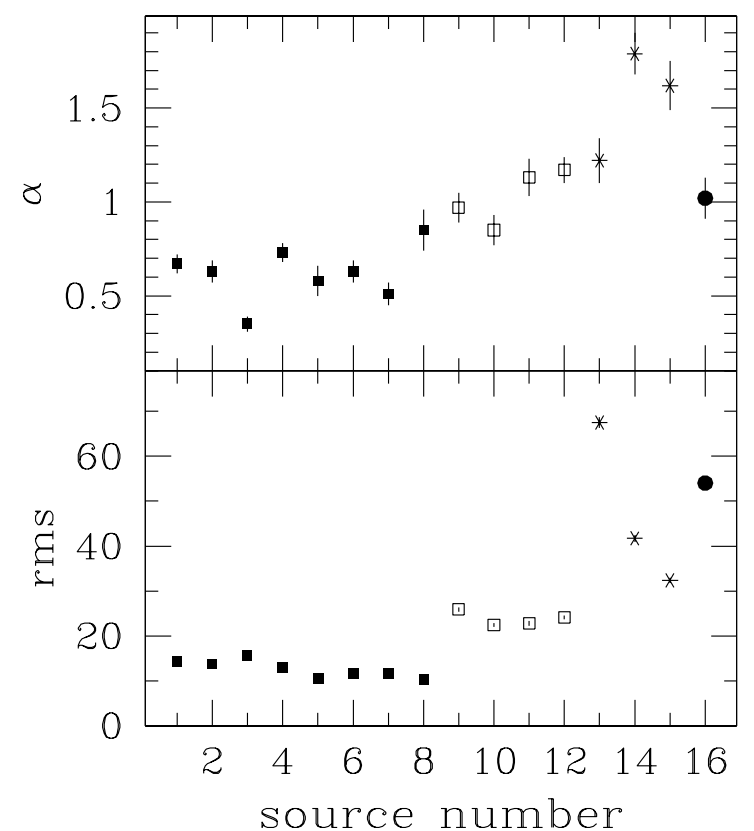

Fig. 4. rms amplitude of the light curves and power-law index of the power spectra of the individual sources (see Table 1). Type I sources are represented by filled squares, type II sources by open squares and type III sources by stars. The filled circle corresponds to Cyg X-1. The errors in rms amplitude are smaller than the symbols.

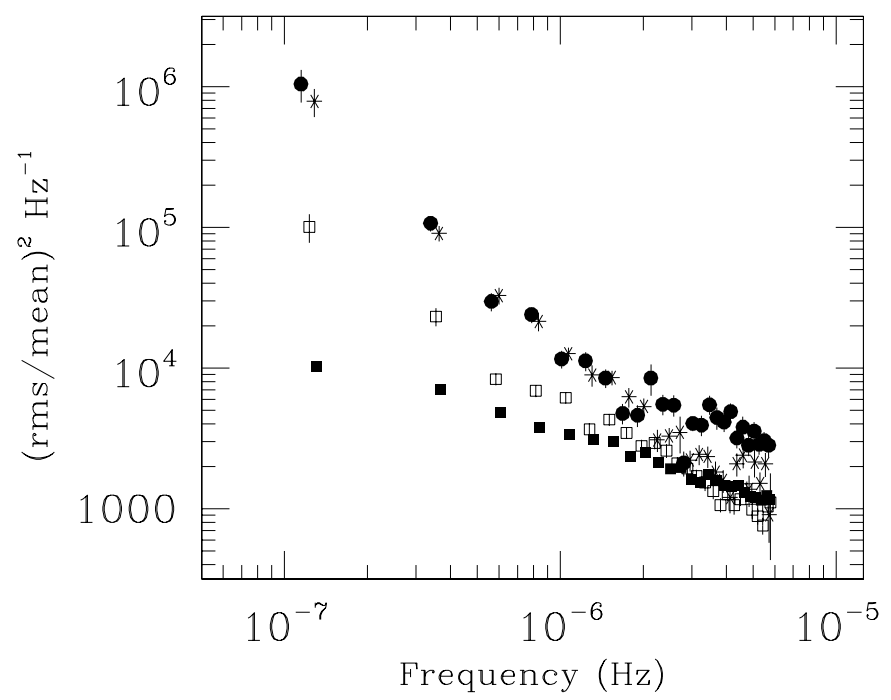

Fig. 5. Comparison of the power spectra of low-mass X-ray binaries with that of Cyg X-1. The power spectra are averaged spectra of the individual sources that belong to each group. Errors are absorbed by the size of the points. Symbols are as in Fig. 4.

be separated into three groups according to the shape of their power density spectra at very low frequencies and the pattern of the long-term X-ray variability of their light curves. As classifying parameters we have taken $i$ ) the fractional amplitude of variability rms from the light curves, $i i)$ the slope $\alpha$ of the bestfit power law from the power spectra and, in a more qualitative way, iii) the shape of the intensity distribution functions.

Type I sources show lower rms and flatter power spectra (rms $<20 \%, \alpha<0.9)$ than type II sources $(20 \%<\mathrm{rms}<30 \%$,

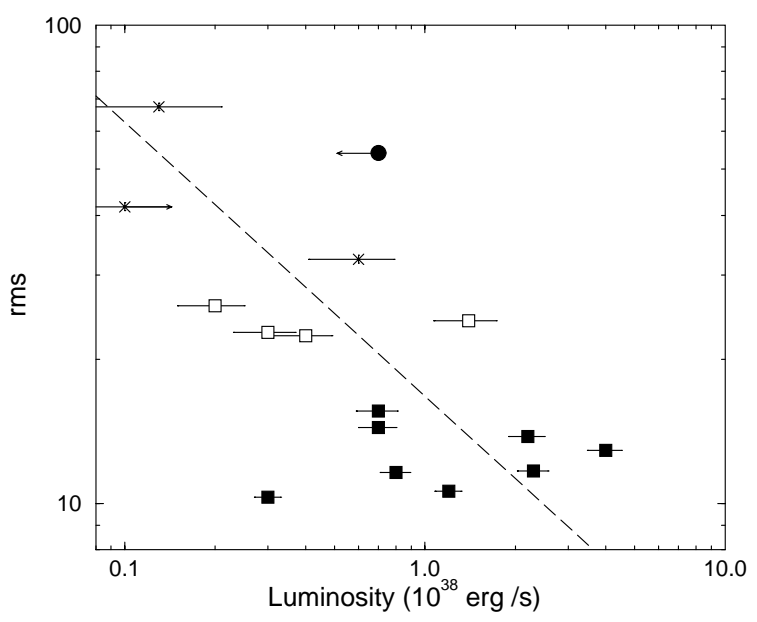

Fig. 6. Fractional amplitude of variability (rms) as a function of $\mathrm{X}$-ray luminosity in the energy range $0.5-20 \mathrm{keV}$, except for the points marked with an arrow, GX354 and Cyg X-1, for which 1-10 keV and 1-30 keV bandwidths apply, respectively. The dashed line represents the best fit to the data using the least-squares bisector line method. Symbols are as in Fig. 4.

$0.9 \lesssim \alpha \lesssim 1.2$ ), and these in turn, have lower rms and flatter power spectra than type III ( $\mathrm{rms}>30 \%, \alpha>1.2$ ). The intensity distribution functions of type I sources tend to show positive tails and are narrower than those of type II sources, which tend to show negative tails. In type III systems the intensity distribution functions are even wider.

It should be noted that the use of just one of these parameters does not generally suffice to characterise unequivocally a system. It is the combination of the spectral and timing parameters together with the extra support from the characteristic shape of the intensity distribution function which allows a more robust way of characterising LMXBs. For example, the slope of the PDS in GX9+9 is similar to those found in Type II sources. However, the rms is about 10 sigmas smaller, more in accordance with type I sources as its IDF is. The light curve exhibits a long-term modulation similar to but lower in amplitude than those of type II systems. Likewise, the power-law index of Cyg X-2 is consistent with the type III source 4U 1705-44 but its rms amplitude and shape of the IDF resemble those of type II sources.

Several schemes have been put forward for the classification of LMXBs: Parsignault \& Grindlay (1978) separated LMXBs into class I and II depending on whether or not a direct correlation between the source temperature (obtained by fitting an exponential to the energy spectrum) and the source intensity exists. Ponman (1982) distinguished between subcritical and supercritical LMXBs depending on whether or not the X-ray luminosity of the system is below or above a certain critical luminosity, approximately half the Eddington limit. White \& Mason (1985) classified LMXBs according to the number of components needed to fit their energy spectra, hence distinguishing between one-component (a single power law with an exponential cutoff) and two-component (also including a blackbody function) systems. Based on the age, the location in the Galaxy and whether or not a low-energy blackbody component was required to fit the energy spectra 
Naylor \& Podsiadlowski (1993) divided the LMXBs into bulge and disc sources. According to the pattern that the source traces out in the colour-colour X-ray diagram and the type of noise components in the $10^{-2}-10^{2} \mathrm{~Hz}$ power density spectra, Hasinger \& van der Klis (1989) categorised LMXBs into Z and atoll sources. Note that the resulting number of different types is always two.

In general, the agreement between these classification schemes is good (see Col. 4 in Table 1). One group would contain Parsignault \& Grindlay's class I, Ponman's supercritical, White \& Mason's two-component spectra, Naylor \& Podsiadlowski's bulge and Hasinger \& van der Klis' Z sources. The other group would include class II, subcritical, onecomponent, disc, atoll sources. Nevertheless, each scheme has some controversial sources that detach from the norm. For example, GX349+2 (Kuulkers \& van der Klis 1998) and GX13+1 (Homan et al. 1998) exhibit both $\mathrm{Z}$ and atoll characteristics. GX9+9 has a much shorter orbital period than the rest of its group (bulge sources in the Naylor \& Podsiadlowski's scheme) making it very unlikely that this system contains a subgiant secondary as has been proposed for the bulge sources (Bandyopadhyay et al. 1999). GX340+0 did not easily fit in Ponman's scheme (Ponman 1982), showing characteristics of both, subcritical and supercritical sources.

When the properties of the long-term variability are taken into account the classification of LMXBs based on the properties of the aperiodic variability at higher frequencies is mixed up. For example, Cyg X-2, which is considered as the prototype of $\mathrm{Z}$ sources, shows a much steeper power spectral continuum $(\alpha=1.2)$ and higher amplitude of variability $(\mathrm{rms}=24 \%)$ than the rest of the $\mathrm{Z}$ sources, whose power-law index and rms distribute around values 0.7 and 13\%, respectively. Likewise, atoll sources do not seem to form an homogeneous group either. Among the atoll sources the power-law index and rms variability vary over a wide range from 0.6 to 1.6 and $10-60 \%$, respectively. Some atoll sources such as GX13+1 or GX9+1 are more similar to $\mathrm{Z}$ sources than to other members of the atoll group.

It might very well be that the classification of LMXBs in terms of a discrete number of groups is an oversimplification and that LMXBs constitute an homogeneous group of sources in which some unknown parameters change continuously to give rise to their differences.

One of these parameters could be the mass accretion rate. The increase of the X-ray luminosity from type III to type I objects may be interpreted as an increase in the accretion rate, probably due to the different nature of the secondary. It was already pointed out by Hasinger \& van der Klis (1989) that the differences between $\mathrm{Z}$ and atoll sources may be due to the different luminosity class of the mass-donating stars, with $\mathrm{Z}$ sources having evolved secondaries and atoll sources main-sequence stars. Infrared spectroscopic measurements (Bandyopadhyay et al. 1999) show evidence indicating that not only the luminosity class but also the spectral type might be different so that $\mathrm{Z}$ sources would contain evolved and earlier than G5 stars and atoll sources late-type ( $\mathrm{K}$ or $\mathrm{M})$, either evolved or main-sequence, stars. Since the mass-loss rate scales with luminosity and radius (Reimers 1975), it is natural to expect that the mass accretion rate will be higher in systems which have an evolved star as an optical companion (like the type I systems).

If the X-ray variations are caused by variations of the accretion rate which propagate toward the inner regions of the accretion disc, then in type I systems, the accretion disc should be able to transfer even the fastest of these variations (faster even than the diffusion time-scale at the outer part of the disc). This can be achieved if the accretion disc is very small in size (perhaps the inner part is missing due to the presence of a relatively strong magnetosphere), or if it is geometrically thick (e.g. Churazov et al. 2001).

As the accretion rate decreases, the disc becomes geometrically thin, allowing only the slowest (and probably largest amplitude) mass-rate variations to propagate toward the inner part. This could explain the very slow modulation in the light curves of type II objects. Type III objects could simply be those type II objects which have: a) the closest companion (note that the only value of the orbital period known for a type III system is much shorter than any other system, see Table 1) and b) the weakest magnetic field. In this case, if the accretion disc is indeed smaller in size (due to the proximity of the companion), then higher frequency oscillations could also propagate from the outer boundary toward the inner parts of the disc. At the same time, the larger amplitude mass accretion rate variations could penetrate the weak magnetosphere and extend all the way to the surface of the neutron star, giving rise to the flaring appearance of their light curves.

This scenario could also explain the dependence of rms on X-ray luminosity that we observe (Fig. 6). Suppose that the observed variations are indeed caused by variations of the mass accretion rate. These variations could be thought of as "flares", which originate at the outermost part of the disc and propagate toward the innermost regions. Suppose now that the shape and size of the flares are fixed in all systems, and that only the number of flares per unit time, say $N$, differ among the various systems. In this case, assuming there is not a significant constant, underlying component, $L_{\mathrm{X}} \propto N$, while rms $\propto N / L_{\mathrm{X}}^{2}$, and so $\mathrm{rms} \propto L_{\mathrm{X}}^{-1}$, inconsistent with the observed relation $\mathrm{rms} \propto L_{\mathrm{X}}^{-0.6}$. Therefore, both $N$ and the typical flare size scale (i.e. duration) should change with luminosity. If, as we move from type I to type II and type III objects, only the longest amplitude variations propagate in the disc (i.e. $N$ decreases) and these variations last longer (i.e. the flare size scale increases), then a relationship between rms and $L_{\mathrm{X}}$, similar to the one shown in Fig. 6 could be expected.

It is worth making a comparison between Cyg $\mathrm{X}-1$ and neutron-star systems. The power spectra of type I sources are significantly different from that of Cyg X-1 both in slope and variability. Type II sources, although they exhibit similar noise shape, are less variable than Cyg $\mathrm{X}-1$. In type III sources the rms is comparable to that of Cyg $\mathrm{X}-1$ but have a steeper slope in the power spectra. The differences between the properties of Cyg X-1 and some of the LMXB such as 4U 1705-44 (whose power-law index and rms amplitude are similar to those of Cyg X-1) are smaller than between some LMXBs themselves. We conclude that the physical processes that cause the lowfrequency X-ray variations in X-ray binaries are determined by 
the conditions of matter in the surroundings of the compact object rather than by its nature (neutron star or black hole).

\section{Conclusions}

Based on the properties of the long-term aperiodic variability we have divided low-mass X-ray binaries into three categories. Systems in each group distinguish themselves by the shape and amplitude of variability of their power density spectra, light curves and intensity distribution functions. It seems plausible that type I systems harbour evolved and possibly early type companions, whereas type II and III systems contain later type main-sequence stars. The mass loss of the companions increases from type III to type II to type I systems, hence the corresponding increase in luminosity. Differences in the accretion disc structure (thin vs. thick) could explain the lack of fast variations in type II systems. Type III systems could also be affected by the weak magnetic field, and perhaps the small size of the disc. Our results imply that, after all, it is not just the accretion physics that dictates the observed characteristics of the systems. Other factors, like the size of the disc and/or the presence of weak magnetic fields, probably affect the low frequency $\mathrm{X}$-ray variations in these systems. Although the separation of LMXBs into these three groups is useful for the purpose of investigating their general aperiodic properties, it is likely that LMXBs constitute an homogeneous group of sources, whose properties vary in a continuous, rather than a discrete, way. Whether the differences in the aperiodic variability between Cyg $X-1$ and LMXBs are due to the different nature of the primary star - a B supergiant in opposition to late G-K type stars - or intrinsic variations in the vicinity of the compact star is unknown. It would be interesting to investigate the long-term variability of black-hole systems with late-type companions. However, owing to the transient nature of these systems such studies are very difficult to perform.

Acknowledgements. Data provided by the ASM RXTE teams at MIT and at the RXTE SOF and GOF at NASA's GSFC. PR acknowledges partial support from the European Union via the Training and Mobility of Researchers Network Grant ERBFMRX/CT98/0195, from the Generalitat Valenciana via the Programme Ayudas para las acciones de apoyo a la investigación. PR is a researcher of the programme Ramón y Cajal funded by the University of Valencia and the Spanish Ministery of Science and Technology.

\section{References}

Bandyopadhyay, R. M., Shahbaz, T., Charles, P. A., \& Naylor, T. 1999, MNRAS, 306, 417

Belloni, T., \& Hasinger, G. 1990, A\&A, 230, 103

Belloni, T., Mendez, M., van der Klis, M., et al. 1996, ApJ, 472, L107

Belloni, T., Psaltis, D., \& van der Klis, M. 2002, ApJ, 572, 392

Christian, D. J., \& Swank, J. H. 1997, ApJS, 109, 177

Churazov, E., Gilfanov, M., \& Revnivtsev, M. 2001, MNRAS, 321, 759

Cowley, A. P., Schmidtke, P. C., Crampton, D., Hutchings, J. B., \& Bolte, M. 1991, ApJ, 373, 228

Fender, R. P., \& Hendry, M. A. 2000, MNRAS, 317, 1

Hasinger, G., \& van der Klis, M. 1989, A\&A, 225, 79

Homan, J., van der Klis, M., Wijnands, R., Vaughan, B., \& Kuulkers, E. 1998, ApJ, 499, L41

Isobe, T., Feigelson, E. D., Akritas, M. G., \& Babu, G. J. 1990, ApJ, 364, 104

Kong, A. K. H., Charles, P. A., \& Kuulkers, E. 1998, NewA, 3, 301

Lwein, W. H. G., \& van Paradijs, J. 1985, A\&A, 149, L27

Liu, Q. Z., van Paradijs, J., \& van den Heuvel, E. P. J. 2001, A\&A, 368,1021

Miyamoto, S., Kimura, K., Kitamoto, S., Dotani, T., \& Ebisawa, K. 1991, ApJ, 383, 784

Naylor, T., \& Podsiadlowski, Ph. 1993, MNRAS, 262, 929

Parsignault, D. R., \& Grindlay, J. E. 1978, ApJ, 225, 970

Ponman, T. 1982, MNRAS, 200, 351

Press, W. H. 1978, Comments on Astrophysics, vol. 7, No. 4, 103

Press, W. H., Teukolsky, S. A., Vetterling, W. T., \& Flannery, B. P. 1992, in Numerical Recipes (Cambridge University Press), 637

Reig, P., Papadakis, I., \& Kylafis, N. 2002, A\&A, 383, 202

Reimers, D. 1975, Mem. Soc. Roy. Sci. Liège, 6e Ser. 8, 369

Schulz, N. S., Hasinger, G., \& Trümper, J. 1989, A\&A, 225, 48

Shahbaz, T., Smale, A. P., Naylor, T., et al. 1996, MNRAS, 282, 1437

Smale, A. P., \& Lochner, J. C. 1992, ApJ, 395, 582

van der Klis, M. 1994a, ApJS, 92, 511

van der Klis, M. 1994b, A\&A, 283, 469

van der Klis, M. 1995, in X-ray Binaries, ed. W. H. G. Lewin, J. van Paradijs, \& E. P. J. van den Heuvel (Cambridge University Press), 252

van der Klis, M. 2000, ARA\&A, 38, 717

White, N. E., \& Mason, K. O. 1985, SSRv, 40, 167

White, N. E. 1989, A\&ARv, 1, 85

Wijnands, R., \& van der Klis, M. 1999, ApJ, 514, 939 